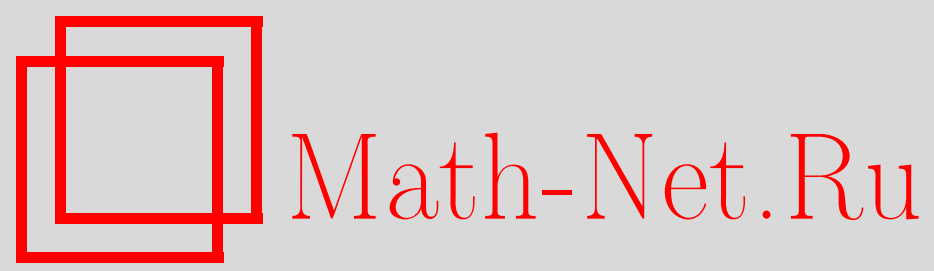

А. Н. Катулев, М. Ф. Малевинский, Методы и алгоритмы повышения качества слабоконтрастного 2D изображения, обнаруживаемого оптико-электронным прибором динамического объекта, Вестник ТвГУ. Серия: Прикладная математика, 2018, выпуск 3, 95-111

DOI: https://doi.org/10.26456/vtpmk512

Использование Общероссийского математического портала Math-Net.Ru подразумевает, что вы прочитали и согласны с пользовательским соглашением

http://www.mathnet.ru/rus/agreement

Параметры загрузки:

IP: 54.224 .187 .69

26 апреля 2023 г., $11: 14: 50$ 


\section{СИСТЕМНЫЙ АНАЛИЗ, УПРАВЛЕНИЕ И ОБРАБОТКА ИНФОРМАЦИИ}

УДК 004. 932.2.517. 968

МЕТОДЫ И АЛГОРИТМЫ ПОВЫШЕНИЯ КАЧЕСТВА

СЛАБОКОНТРАСТНОГО 2D ИЗОБРАЖЕНИЯ, ОБНАРУЖИВАЕМОГО ОПТИКО-ЭЛЕКТРОННЫМ ПРИБОРОМ ДИНАМИЧЕСКОГО ОБЪЕКТА

Катулев А.Н.*, Малевинский М.Ф.**

*Научно-исследовательский центр Центрального НИИ войск ВКО Минобороны

России, г. Тверь

**Тверской государственный университет, г. Тверь

Поступила в редакцию 23.01.2018, после переработки 05.09.2018.

Для повышения качества слабоконтрастного 2D изображения, формируемого оптико-электронным прибором по обнаруживаемому динамическому объекту, предложены методы и алгоритмы повышения яркости сложением в частотной области вейвлет-спектров накапливаемых изображений на отрезке времени обнаружения объекта. Суммарное изображение восстанавливается обратным вейвлет-преобразованием. Вейвлетспектры являются достаточными статистиками, вычисляются по системе вейвлетов, построенной на основе волновой вытянутой сфероидальной функции нулевого порядка. Предложены методы и алгоритмы фильтрации на основе принципа разделения вейвлет-спектров изображения динамического объекта, излучения фона в зоне контроля прибора и шума прибора, а также повышения контраста интегральным оператором дифференцирования.

Ключевые слова: оптико-электронный прибор, динамический объект, слабоконтрастное $2 \mathrm{D}$ изображение, статистика повышения яркости, алгоритм, показатели эффективности.

Вестник ТвГУ. Серия: Прикладная математика. 2018. № 3. С. 95-111. https://doi.org/10.26456/vtpmk512

\section{Введение}

Аксиоматическими истинами являются утверждения, что

- слабоконтрастные 2D изображения формируются оптико-электронным прибором по обнаруживаемым ДО-динамическим объектам (здесь и ниже рассматриваются только воздушные объекты), находящимся на таком удалении, при котором существует малое оптическое излучение объекта по отношению 
к излучению атмосферного фона, либо по ДО, находящимся в облаках, либо совершающим вираж (установившийся или неустановившийся), при котором оптическое излучение в направлении оптико-электронного прибора-ОЭП осуществляется малой излучательной поверхностью объекта по отношению к излучению атмосферного фона,

- 2D изображение, формируемое ОЭП по обнаруживаемому объекту при априорной неопределенности о текущей фоноцелевой обстановке даже в простых условиях функционирования, является реализацией случайного двумерного процесса, которому свойственны нестационарности: скачки, острые пики и перепады,

- повышение яркости слабоконтрастного изображения, получаемого в текущих условиях функционирования ОЭП, возможно лишь при условии накопления реализаций изображения и их «когерентного» сложения (то есть при полной совмещенности реализаций) либо при условии априори знания характеристики передачи уровней изображения [1-3] и что

- повышение яркости каждого элемента изображения может приводить к повышению контраста (резкости) изображения, а значит, к повышению вероятности правильного распознавания типа обнаруживаемого ДО и к устойчивости его сопровождения ОЭП.

Необходимость правильного распознавания типа ДО и устойчивого его сопровождения обусловливает, в свою очередь, актуальность повышения яркости слабоконтрастного 2D изображения обнаруживаемого объекта.

Процедура повышения яркости изображения реализуется операцией сложения накопленных изображений на отрезке времени обнаружения объекта. Сложение изображений возможно в пространственно-временной области и в пространственно-частотной. Обеспечение полной совмещенности-«когерентности» слагаемьх изображений ДО при этом принципиально возможно только при установлении на них устойчивых одних и тех же реперных элементов.

Устойчивые реперные элементы устанавливаются очевидным образом в частотно-временной области, а в пространственно-временной из-за движения объекта и изменения по неизвестному закону его излучательной поверхности, например, при маневре объекта, а также из-за случайности реализаций изображения и их особенностей, связанных с возможными скачками и острыми пиками, требование их (реперных элементов) устойчивости практически не реализуемо без введения априорных данных. Однако последних не имеется.

Отметим также, что априорных данных о формировании характеристики передачи уровней изображения как необходимого условия [1-3] одного из методов повышения качества изображения в пространственно-временной области не имеется в текущих условиях обнаружения объекта: текущая фоноцелевая обстановка в зоне контроля прибора нестационарна и априори неизвестна. Не имеется в настоящее время и общей теории улучшения изображений (в том числе повышения яркости). Разработаны отдельные приемы улучшения изображений. K основным из них можно отнести [1-4]: 
- преобразование яркости посредством коррекции амплитудных характеристик, линейного повышения контраста, преобразования гистограмм, пороговой обработки, препарирования,

- градационные преобразования, усреднение изображений,

- выделение контуров операторами дифференцирования: градиентным, Робертса, Лапласа,

- пространственная линейная и нелинейная фильтрация,

- оценка геометрических характеристик изображения.

В [2] предложены также критерии качества изображений: критерий визуального восприятия, среднеквадратический, максимальной ошибки, вероятностнозональный.

Однако применение предложенных приемов и критериев непосредственно не распространено на решение задачи улучшения качества изображений ДО, обнаруживаемого на последовательности фоноцелевых кадров со слабоконтрастными изображениями, а для реализации критериев требуется априорная информация об идеальном изображении обнаруживаемого объекта.

Поэтому повышение яркости слабоконтрастного 2D изображения, формируемого ОЭП по обнаруживаемому ДО на конечном отрезке времени при априорной неопределенности о текущей фоноцелевой обстановке, практически возможно и целесообразно осуществлять только в пространственно-частотной области и с использованием вейвлет-преобразования. Последнее для рассматриваемого случая более предпочтительно по сравнению с Фурье-преобразованием.

Предпочтительность обусловлена известными недостатками Фурьепреобразования. Так [5], Фурье-преобразование слабо реагирует на скачки, разрывы и острые пики реализаций изображения, а возникающие при этом частотные изменения «размазывает» по всем частотам, ему свойствен эффект Гиббса в окрестностях скачков и разрывов в реализациях, Фурье-преобразование не дает представления о локальных свойствах реализаций при быстрых временных изменениях их спектрального состава. В результате применения Фурье-преобразования в изображение будут внесены искажения.

Вейвлет-преобразование свободно от подобных недостатков [6,7]. Для решения задачи повышения яркости изображения ниже применяется вейвлет, синтезированный на основе волновой вытянутой сфероидальной функции нулевого порядка. Доказано [8,9], что такой вейвлет доминирует над другими часто применяемыми в практике исследования случайных процессов вейвлетами.

\section{1. Цель статьи}

Разработка алгоритма повышения яркости и контраста 2D изображения воздушного динамического объекта, обнаруживаемого на последовательности слабоконтрастных фоноцелевых кадров оптико-электронного прибора.

Оценка характеристик качества алгоритма. 


\section{2. Постановка задачи, принцип решения}

Содержательно научная задача заключается в синтезировании преобразования суммы несовмещенных (не «когерентных») слабоконтрастных 2D изображений, формируемых ОЭП в виде пространственно-временной последовательности по обнаруживаемому ДО, в сумму совмещаемых («когерентных») пространственночастотных спектров последовательности слабоконтрастных изображений с последующим переходом от суммарного спектра к пространственно-временному 2D изображению с попиксельно повышенным качеством - яркостью относительно качества - яркости каждого слабоконтрастного 2D изображения исходной последовательности.

В математических терминах задача формулируется на основе теоремы Планшереля $\left[13\right.$, с. 455]. Утверждается, что линейные двумерное пространство $L_{2}$ интегрируемых с квадратом изображений отображается на пространство $l_{2}$ вейвлет-коэффициентов (коэффициентов Фурье) линейно, значит имеется взаимно однозначное соответствие между суммой изображений и суммой их вейвлеткоэффициентов.

Отображение удовлетворяет равенству Парсеваля и, как следствие, имеет место изоморфизм, т.е. отображение имеет обратное отображение. При этом каждому соответствует своя частота, совокупность которых представляет пространственно- частотный спектр изображения.

Для сложения таких спектров, очевидно, не требуется введения реперных точек - ими являются одинаковые частоты спектров, а результат их сложения, естественно, приведет к повышению яркости суммарного изображения обнаруживаемого динамического объекта.

Покажем равнозначность перехода из пространственно-частотной области и обратно суммирования кадров изображений. Эти преобразования выполним для функций от двух аргументов.

$\mathrm{C}$ помощью вейвлета $\psi(x)$ и $\varphi(y)$ масштабирующей функции для двумерного вейвлет - преобразования аппроксимируем изображение кадра $f(x, y)$ в виде [7]

$$
f(x, y)=\sum_{k, n=-\infty}^{\infty} c_{1 k n} a_{1 k n}(x, y)+c_{2 k n} a_{2 k n}(x, y)+c_{3 k n} a_{3 k n}(x, y)+c_{4 k n} a_{4 k n}(x, y)
$$

где $c_{i k n}=\left(f, a_{i k n}\right), \quad i=1, \ldots, 4-$ вейвлет-коэффициенты (коэффициенты Фурье) функции $f(x, y)$ по координатным функциям $a_{i k n}(x, y)$, $i=1, \ldots, 4 ; \mathrm{n}, \mathrm{k}=0, \pm 1, \ldots$

$$
\begin{aligned}
& \left.\left.a_{1 k n}(x, y)=\varphi_{k}(x) \varphi_{n}(y)=\frac{1}{m} \varphi((x-k) / m)\right) \varphi((y-n) / m)\right), \\
& \left.\left.a_{2 k n}(x, y)=\varphi_{k}(x) \varphi_{n}(y)=\frac{1}{m} \varphi((x-k) / m)\right) \psi((y-n) / m)\right), \\
& \left.\left.a_{3 k n}(x, y)=\psi_{k}(x) \varphi_{n}(y)=\frac{1}{m} \psi((x-k) / m)\right) \varphi((y-n) / m)\right), \\
& \left.\left.a_{4 k n}(x, y)=\psi_{k}(x) \psi_{n}(y)=\frac{1}{m} \psi((x-k) / m)\right) \psi((y-n) / m)\right)
\end{aligned}
$$


двумерные сепарабельные вейвлеты, где $m$ - параметр масштаба, $k, n$ - параметры сдвига.

Преобразование Фурье (спектр) функции $f$ равно

$$
\begin{aligned}
S_{f}\left(\omega_{x}, \omega_{y}\right) & =\sum_{k, n=-\infty}^{\infty} c_{1 k n} S_{1 k n}\left(\omega_{x}, \omega_{y}\right)+c_{2 k n} S_{2 k n}\left(\omega_{x}, \omega_{y}\right)+ \\
& +c_{3 k n} S_{3 k n}\left(\omega_{x}, \omega_{y}\right)+c_{4 k n} S_{4 k n}\left(\omega_{x}, \omega_{y}\right)
\end{aligned}
$$

где

$$
\begin{aligned}
& S_{1 k n}\left(\omega_{x}, \omega_{y}\right)=S_{\varphi k}\left(\omega_{x}\right) S_{\varphi n}\left(\omega_{y}\right) / m, \\
& S_{2 k n}\left(\omega_{x}, \omega_{y}\right)=S_{\varphi k}\left(\omega_{x}\right) S_{\psi n}\left(\omega_{y}\right) / m, \\
& S_{3 k n}\left(\omega_{x}, \omega_{y}\right)=S_{\psi k}\left(\omega_{x}\right) S_{\varphi n}\left(\omega_{y}\right) / m, \\
& S_{4 k n}\left(\omega_{x}, \omega_{y}\right)=S_{\psi k}\left(\omega_{x}\right) S_{\psi n}\left(\omega_{y}\right) / m
\end{aligned}
$$

спектры функций формулы (1).

Здесь предполагается, что в прямом и обратном преобразовании Фурье присутствует множитель $1 /(2 \pi)$.

Аппроксимируем теперь в частотной области спектральную функцию $S_{f}\left(\omega_{x}, \omega_{y}\right)$ по системе функций

$$
\begin{gathered}
S_{l k n}\left(\omega_{x}, \omega_{y}\right), l=1-4, n, k=0, \pm 1, \ldots \\
S_{f}\left(\omega_{x}, \omega_{y}\right)=\sum_{l=1}^{4} \sum_{k, n=-\infty}^{\infty} b_{l k n} S_{l k n}\left(\omega_{x}, \omega_{y}\right),
\end{gathered}
$$

где $b_{l k n}=\left(S_{f}, \bar{S}_{l k n}\right)$, знак - означает комплексное сопряжение.

Из равенства Парсеваля $[5$, с. $55-56]\left(S_{f}, \bar{S}_{l k n}\right)=\left(f, \bar{a}_{l k n}\right)$ следует, что

$$
c_{l k n}=b_{l k n}, k=0, \pm 1, \ldots, l=1-4
$$

Взяв обратное преобразование Фурье от функции (3), на основании (4), получим функцию $f(x, y)$.

Просуммировав $N$ кадров изображений $f_{1}, \ldots f_{N}$, получим

$$
\sum_{l=1}^{N} f_{l}(x, y)=\sum_{l=1}^{4} \sum_{k, n=-\infty}^{\infty} c_{l k n} a_{l k n}(\mathrm{x}, \mathrm{y}) .
$$

Просуммируем спектры $S_{f l}\left(\omega_{x}, \omega_{y}\right), l=1,2, \ldots, N$

$$
\sum_{l=1}^{N} S_{f l}\left(\omega_{x}, \omega_{y}\right)=\sum_{l=1}^{4} \sum_{k, n=-\infty}^{\infty} b_{l k n} S_{l k n}(\omega) .
$$

Из (4)-(6) следует, что суммирование кадров изображений равнозначно суммированию их спектров.

Ниже будем вычислять спектры $S_{1}\left(\omega_{x}, \omega_{y}\right), S_{2}\left(\omega_{x}, \omega_{y}\right)$ кадров изображений с использованием двумерного вейвлет-преобразования. 
Для фильтрации шумов на изображении используется принцип разделения его спектра на вейвлет-спектр изображения собственно динамического объекта и вейвлет-спектр излучения фона в зоне контроля прибора и шума прибора.

Справедливость разделения непосредственно исходит и из известного, например [10], факта: в спектре изображения собственно динамического объекта содержатся более низкие частоты, чем в спектре фона и шума. Отсюда, как следствие, утверждается возможность программно фильтровать фоновую помеху и шум, а затем восстанавливать в пространственно-временной области обратным двумерным вейвлет-преобразованием суммарное изображение с повышенной яркостью в пространственно-частотной области.

Итак, задача повышения яркости изображения обнаруживаемого ОЭП динамического объекта сводится:

- к задаче вычисления $S_{1}\left(\omega_{x}, \omega_{y}\right), S_{2}\left(\omega_{x}, \omega_{y}\right)$ - прямьх вейвлет-преобразовний функций яркости $f_{1}(x, y)$ и $f_{2}(x, y)$ исходных последовательно формируемых ОЭП изображений,

- фильтрации из $S_{1}\left(\omega_{x}, \omega_{y}\right), S_{2}\left(\omega_{x}, \omega_{y}\right)$ вейвлет-спектров $S_{1 ф ш ~}\left(\omega_{x}, \omega_{y}\right)$, $S_{2 \phi ш}\left(\omega_{x}, \omega_{y}\right)$ фона $(\phi)$ в зоне контроля ОЭП и шума (ш) прибора, для этого используется функция очистки от шумов изображения системы MatLab,

- почастотному сложению вейвлет-спектров $S_{1 д о}\left(\omega_{x}, \omega_{y}\right), S_{2 \text { до }}\left(\omega_{x}, \omega_{y}\right)$ изображений собственно обнаруживаемого прибором динамического объекта (до), формированию $f_{1,2, \text { до }}(x, y)$ изображения объекта в пространственновременной области посредством обратного вейвлет-преобразования суммарного вейвлет-спектра $S_{1,2 д о}\left(\omega_{x}, \omega_{y}\right)=S_{1 д о}\left(\omega_{x}, \omega_{y}\right)+S_{2 д о}\left(\omega_{x}, \omega_{y}\right)$;

- к оценке качества изображения $f_{1,2}$, до $(x, y)$ по показателям яркости, контраста и фильтрации фона и шума.

Такая формулировка задачи по существу содержит и принцип ее решения принцип сводится к последовательно попарному сложению изображений при любой длине последовательности слабоконтрастных изображений. Так, при сложении трех последовательных изображений исходными являются функция яркости $f_{1,2, \text { до }}(x, y)$, как результат сложения первых двух изображений, и функция яркости $f_{3}(x, y)$ - третьего изображения. Аналогично выполняется сложение четырех, пяти и т.д. до $n$-го последовательного изображения, т.е. до получения суммарного изображения $f_{1, . ., n, \text { до }}(x, y)$ по последовательности из $n$ слабоконтрастных изображений.

\section{3. Принцип повышения контраста}

Контраст суммарного изображения будем повышать с использованием [11] двумерного интегрального оператора дифференцирования со специально выбранным ядром типа «синус $x$ на $x \gg$ при аппроксимации производной двумерной дискретной функции яркости суммарного изображения функционалом вида

$$
f\left(a_{x}, a_{y}, b_{x}, b_{y}\right)=\frac{1}{\sqrt{a_{x} a_{y}}} \int_{-T_{x}}^{T_{x}} \int_{-T_{y}}^{T_{y}} \frac{d}{d x} \frac{\sin \left[\left(x-b_{x}\right) / a_{x}\right]}{\pi\left(x-b_{x}\right)} \cdot \frac{d}{d y} \frac{\sin \left[\left(y-b_{y}\right) / a_{y}\right]}{\pi\left(y-b_{y}\right)} f_{1, . ., n, \text { ДО }}(x, y) d x d y
$$


где $\mathrm{T}_{\mathrm{X}}, \mathrm{T}_{Y}$ - заданные значения правых границ симметричных интервалов по системе координат $x$ и $y$ соответственно апертуры (маски) оператора.

Введенный интегральный оператор дифференцирования обеспечивает вычисление производных изображения с подавлением в них высокочастотных составляющих. Из этого оператора вытекают, как частные, известные операторы дифференцирования изображений - операторы Лапласа, Робертса, градиентный [11].

\section{4. Алгоритм решения задачи в целом}

Включает алгоритм вычисления вейвлет-масштабирующей и вейвлет-функций на основе волновой вытянутой сфероидальной функции нулевого порядка, алгоритм повышения яркости слабоконтрастного изображения ДО и алгоритм повышения контраста изображения.

Необходимость первого из них обусловлена известным обстоятельством [6,7]: двумерное вейвлет-преобразование выполняется на основе тензорного произведения двух одномерных вейвлетов и для этого требуется соответственно иметь масштабирующие и вейвлет-функции.

\section{5. Структура алгоритма вычисления вейвлет-масштабирующей и вейвлет-функций на основе ВВСФ нулевого порядка}

Предварительно отметим, что вычисление ВВСФ - волновой вытянутой сфероидальной функции нулевого порядка осуществляется при ее аппроксимации отрезком ряда Котельникова вида [9]

$$
w(\tau)=\sum_{k=-N}^{N} w_{k}[\sin \pi(\tau-k \Delta) / \Delta] /[\pi(\tau-k \Delta) / \Delta]
$$

где $w_{k}=w(k \Delta), k \in[-N, N]$ - значения решения в точке $k \Delta$ интегрального уравнения Фредгольма второго рода с ядром типа $\operatorname{sinc}(t)=\sin (t) / t, C-$ верхняя граничная частота финитного спектра ВВСФ, $0<\Delta \leq \pi / \mathrm{C}, C_{1}=\pi / \Delta, \Delta-$ шаг дискретности на отрезке $[-T, T], N=[T / \Delta]$ целая часть числа Т $/ \Delta$.

Затем вычисления осуществляются в следующей последовательности [8]:

1. Вычисление коэффициентов $w_{k}, k \in[-N, N]$, как решение задачи полной проблемы собственных значений и собственных векторов с использованием алгоритма [3] в системе MatLab.

2. Вычисление вейвлет-масштабирующей функции

$$
\varphi(t)=(1 / \sqrt{A}) \sum_{r=-N}^{N} w_{r} \sin c\left(C_{1} t-r \pi\right)
$$

где $A=\Delta \sum_{l=-N}^{N} w_{l}^{2}$ нормировочный коэффициент и выше было отмечено $t=x, y$.

3. Вычисление коэффициентов $h_{k}=\sqrt{2} \int_{-T}^{T} \varphi(t) \varphi(2 t-\Delta k) d t, k=-N, \ldots, N$. 
4. Вычисление коэффициентов $g_{k}=(-1)^{k} h_{-k}, k=-N, \ldots, N$.

5. Вычисление собственно вейвлет-ВВСФ нулевого порядка

$$
\psi(t)=\sqrt{2} \sum_{k=-N}^{N} g_{k} \varphi(2 t-\Delta k) .
$$

6. Формирование по масштабирующим функциям $\varphi(x), \varphi(y)$ и вейвлетам $\psi(x)$, $\psi(y)$ порождаемых ими базисных функций в $L^{2}(R)$

$$
\varphi_{m k}(x), \psi_{m k}(x), \varphi_{m n}(y), \psi_{m n}(y)
$$

для последующего формирования разделимых масштабирующей и базисных (по строкам, столбцам и диагоналям) вейвлет-функций в $L^{2}\left(R^{2}\right)$

$$
\varphi_{m k}(x) \varphi_{m k}(y), \quad \varphi_{m k}(x) \psi_{j n}(y), \quad \psi_{m k}(x) \varphi_{m n}(y), \quad \psi_{m k}(x) \psi_{m n}(y)
$$

с целью разложения по последним двумерного изображения $f(x, y)$.

\section{6. Структура алгоритма повышения качества изображения}

Включает следующие операции:

1. Формирование двумерных массивов последовательности слабоконтрастных изображений обнаруживаемого оптико-электронным прибором ДО.

2. Вычисление пространственно-частотных спектров $S_{1}\left(\omega_{x}, \omega_{y}\right), S_{2}\left(\omega_{x}, \omega_{y}\right)$ исходных изображений по функциям их яркости $f_{1}(x, y)$ и $f_{2}(x, y)$ с использованием функции прямого двумерного вейвлет-преобразования.

3. Фильтрация из вычисленных спектров $S_{1}\left(\omega_{x}, \omega_{y}\right), S_{2}\left(\omega_{x}, \omega_{y}\right)$ вейвлетспектров $S_{1 ф ш}\left(\omega_{x}, \omega_{y}\right), S_{2 ф ш}\left(\omega_{x}, \omega_{y}\right)$ - фона $(\phi)$ в зоне контроля оптикоэлектронного прибора и шума (ш) прибора, для этого используется функция очистки от шумов изображения системы MatLab.

4. Почастотное сложение вейвлет-спектров $S_{1 д о}\left(\omega_{x}, \omega_{y}\right), S_{2 д о}\left(\omega_{x}, \omega_{y}\right)$ изображений, очищенных от фоновых помех.

5. Восстановление изображения $f_{1,2, \text { до }}(x, y)$ динамического объекта в пространственно-временной области посредством обратного вейвлетпреобразования вычисленного почастотным сложением вейвлет-спектра

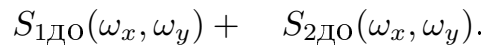

6. Контрастирование границ восстановленного изображения $f_{1,2, \text { до }}(x, y)$ динамического объекта интегральным оператором дифференцирования.

7. Реализация вычислительных операций 2-6 по последовательности из $n$ слабоконтрастных изображений и формирование суммарного изображения $f_{1, . ., n, \text { до }}(x, y)$. 
8. Оценка качества изображения $f_{1, . ., n, \text { до }}(x, y)$ по показателям яркости, фильтрации фоновых помех и шума, а также по показателю контраста.

9. На каждом текущем такте работы алгоритма $n \geq 2$ вычисляется сумма квадратов разностей пикселов восстановленного текущего кадра и предыдущего.

10. Если эта сумма больше заданного числа, то на п.1 для обработки следующего кадра, где в п.2-п.4 суммирование вейвлет-коэффициентов кадра производится с предыдущей суммой.

11. Если сумма квадратов разностей меньше заданного числа, то запоминается номер $\mathrm{N}$ текущего кадра и далее обработка кадров производится на «скользящем» фиксированном интервале в $\mathrm{N}$ кадров.

Вычисления заканчиваются после обработки заданного числа кадров в последовательности.

\section{7. Результаты вычислительных экспериментов}

При сложении изображений ДО, полученных от ОЭП на последовательных слабо контрастных фоноцелевых кадрах, должно быть достигнуто повышение качества изображения обнаруживаемого ДО до уровня качества изображения ДО, обнаруживаемого ОЭП в простых условиях его функционирования.

Вычислительные эксперименты проводились с использованием кадров изображений летательных аппаратов (ДО), снятых любительской видеокамерой (ОЭП) во время демонстрационных полетов на Международном авиационном космическом салоне (МАКС ) в Жуковском в 2017 году.

Результаты вычислительных экспериментов получены для четырех реальных фоноцелевых условий функционирования ОЭП. На рисунках ниже представлены исходные от ОЭП последовательности изображений и результаты их последовательного сложения.

\section{Первая ситуация}

\begin{tabular}{|c|c|c|c|c|c|c|}
\hline Кадры & 1 & 2 & 3 & 4 & 5 & 6 \\
\hline Изображение & & & & & & \\
\hline $\begin{array}{c}\text { Восстановленое } \\
\text { изображение }\end{array}$ & & & & & & \\
\hline $\begin{array}{c}\text { Диференцирование } \\
\text { оператором } \\
\text { Собела }\end{array}$ & s & & 4 & & 3 & \\
\hline
\end{tabular}

На восстановленном изображении ДО будет темнее или светлее, чем на исходном изображении, в зависимости от начального отношения ДО к фону. Фон темнее - ДО будет светлее, фон светлее - ДО темнее.

Показателями качества алгоритма приняты [2]: 


\section{Вторая ситуация}

\begin{tabular}{|c|c|c|c|c|c|c|}
\hline Кадры & 1 & 2 & 3 & 4 & 5 & 6 \\
\hline Изображение & & & & & & \\
\hline $\begin{array}{c}\text { Восстановленое } \\
\text { изображение }\end{array}$ & & & & & & \\
\hline $\begin{array}{c}\text { Диференцирование } \\
\text { оператором } \\
\text { Собела }\end{array}$ & $=L$ L & $\left(\frac{11}{2}\right.$ & $(z)$ & $=c h$ & $C g$ & \\
\hline
\end{tabular}

Третья ситуация

\begin{tabular}{|c|c|c|c|c|c|c|}
\hline Кадры & 1 & 2 & 3 & 4 & 5 & 6 \\
\hline Изображение & & & & & & \\
\hline $\begin{array}{c}\text { Восстановленое } \\
\text { изображение }\end{array}$ & & a & 4 & & & \\
\hline $\begin{array}{c}\text { Диференцирование } \\
\text { оператором } \\
\text { Собела }\end{array}$ & $i$ & id & $?$ & $P_{i}$ & $P_{j}$ & \\
\hline
\end{tabular}

Четвертая ситуация

\begin{tabular}{|c|c|c|c|c|c|c|}
\hline Кадры & 1 & 2 & 3 & 4 & 5 & 6 \\
\hline Изображение & & & & & & \\
\hline $\begin{array}{c}\text { Восстановленое } \\
\text { изображение }\end{array}$ & & & & & & \\
\hline $\begin{array}{l}\text { Диференцирование } \\
\text { оператором Канни }\end{array}$ & & & & . & - & \\
\hline
\end{tabular}


Повышение контраста интегральным оператором дифференцирования Первая ситуация

\begin{tabular}{|c|c|c|c|c|c|c|}
\hline Фоноцелевые кадры & 1 & 2 & 3 & 4 & 5 & 6 \\
\hline $\begin{array}{c}\text { Последовательность } \\
\text { изображений, сфор- } \\
\text { мированных ОЭП }\end{array}$ & & & & & & \\
\hline $\begin{array}{c}\text { Восстановленное } \\
\text { изображение при } \\
\text { последовательном } \\
\text { сложении вейвлет- } \\
\text { спектров }\end{array}$ & & & & & & \\
\hline \multicolumn{6}{|c|}{$\begin{array}{c}\text { Выделенная оператором дифференцирования граница } \\
\text { суммарного изображения по шести ФЦК }\end{array}$} & \\
\hline
\end{tabular}

\section{Вторая ситуация}

\begin{tabular}{|c|c|c|c|c|c|c|}
\hline Фоноцелевые кадры & 1 & 2 & 3 & 4 & 5 & 6 \\
\hline $\begin{array}{c}\text { Последовательность } \\
\text { изображений, сфор- } \\
\text { мированных ОЭП }\end{array}$ & 4 & 6 & a & 8 & a & 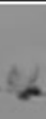 \\
\hline $\begin{array}{c}\text { Восстановленное } \\
\text { изображение при } \\
\text { последовательном } \\
\text { сложении вейвлет- } \\
\text { спектров }\end{array}$ & & 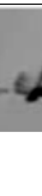 & & & & \\
\hline \multicolumn{6}{|c|}{$\begin{array}{c}\text { Выделенная оператором дифференцирования граница } \\
\text { суммарного изображения по шести ФЦК }\end{array}$} & \\
\hline
\end{tabular}

\section{Третья ситуация}

\begin{tabular}{|c|c|c|c|c|c|c|}
\hline Фоноцелевые кадры & 1 & 2 & 3 & 4 & 5 & 6 \\
\hline $\begin{array}{c}\text { Последовательность } \\
\text { изображений, сфор- } \\
\text { мированных ОЭП }\end{array}$ & 4 & 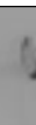 & $\alpha$ & 1 & 4 & \\
\hline $\begin{array}{c}\text { Восстановленное изоб- } \\
\text { ражение при последо- } \\
\text { вательном сложении } \\
\text { вейвлет-спектров }\end{array}$ & & J & S & 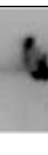 & & \\
\hline \multicolumn{6}{|c|}{$\begin{array}{c}\text { Выделенная оператором дифференцирования граница } \\
\text { суммарного изображения по шести ФЦК }\end{array}$} & \\
\hline
\end{tabular}


Четвертая ситуация

\begin{tabular}{|c|c|c|c|c|c|c|}
\hline Фоноцелевые кадры & 1 & 2 & 3 & 4 & 5 & 6 \\
\hline $\begin{array}{c}\text { Последовательность } \\
\text { изображений, сфор- } \\
\text { мированных ОЭП }\end{array}$ & & & & & & \\
\hline $\begin{array}{c}\text { Восстановленное изо- } \\
\text { бражение при после- } \\
\text { довательном сложе- } \\
\text { нии вейвлет-спектров }\end{array}$ & & & & & & \\
\hline \\
Выделенная оператором дифференцирования граница \\
суммарного изображения по шести ФцК
\end{tabular}

- коэффициент усиления яркости изображения при последовательном сложении слабоконтрастных 2D изображений исходной последовательности;

- коэффициент повышения контраста изображения при последовательном сложении слабоконтрастных 2D изображений исходной последовательности;

- коэффициент фильтрации фоновых помех и шума прибора.

Оценки показателей качества работы алгоритма приведены в таблице 1.

Таблича 1: Показатели качества работы алгоритма

\begin{tabular}{|l|l|}
\hline Показатель (коэффициент) & $\begin{array}{l}\text { Значение показателя, вычисленно- } \\
\text { го по последовательности изобра- } \\
\text { жений из шести кадров ОЭП в } \\
\text { четырех фоноцелевых ситуациях } \\
\text { (рис.1-4) }\end{array}$ \\
\hline Усиления яркости (в \%) & 150330880 \\
\hline Повышения контраста в (\%) & 160350910 \\
\hline Фильтрации помех в (\%) & не менее 200 \\
\hline
\end{tabular}

Вычисление коэффициента фильтрации выполнено при условии независимости слабоконтрастных изображений, однородности и аддитивности случайных фоновых помех и шума прибора и описании [12] энергетической яркости таких помех и шума нормальным законом распределения вероятностей. Поэтому при сложении изображений энергетическая яркость помех и шума уменьшается соответственно количеству $(n)$ складываемых слабоконтрастных изображений (по показателю среднеквадратического отклонения в $\sqrt{n}$ раз).

В таблице 1 к вышеприведенным иллюстрациям представлена динамика повышения показателей в зависимости от количества фоноцелевых кадров, последовательно поступающих от ОЭП. Видно, что имеет место сходимость к контрастным изображениям результатов суммирования слабоконтрастных изображений обнаруживаемых ОЭП динамических объектов в различных фоноцелевых ситуациях. Иллюстрация сходимости дополнительно представлена на Рис. 1, где на оси 
абсцисс обозначены индексы последовательных фоноцелевых кадров со слабоконтрастными изображениями ДО (затрачиваемое время на их получение составляет десятые доли секунды), а на оси ординат - средние арифметические значения яркостей суммарного изображения по каждой ситуации.

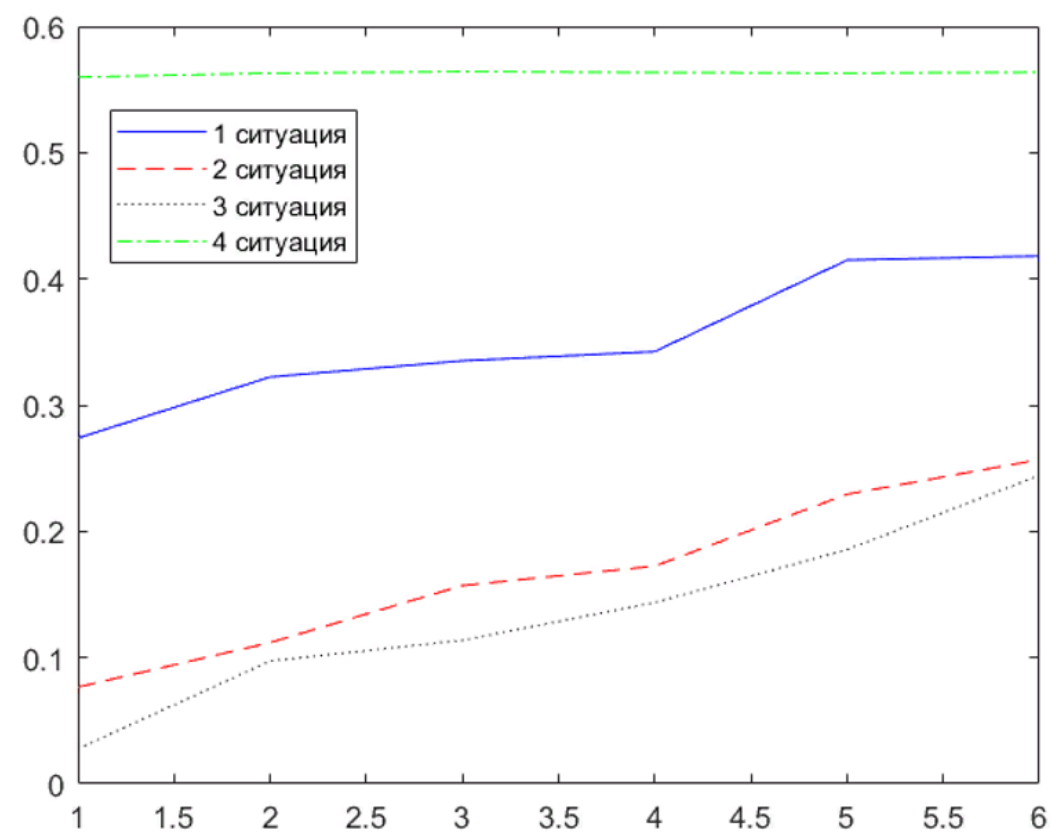

Рис. 1: Результаты работы алгоритма

Из результатов работы алгоритма, представленных на графиках Рис. 1, следует, что требуемое повышение качества достигается при сложении изображений на 2-7 последовательных слабоконтрастных фоноцелевых кадрах.

\section{Заключение}

Построены алгоритмы повышения яркости и контраста изображения, обнаруживаемого ОЭП ДО на последовательности формируемых прибором фоноцелевых кадров-ФЦК со слабоконтрастными изображениями.

Алгоритм инвариантен к различным особенностям фоноцелевой обстановки и полета воздушного ДО, а также к различным выбросам и перепадам яркости на исходных слабоконтрастных изображениях.

Повышение яркости изображения ДО выполнено в пространственно-частотной области с применением вейвлет-преобразования, основанного на вытянутой волновой сфероидальной функции нулевого порядка. Такой вейвлет высокочувствителен к различным особенностям по яркости и контрасту исходных слабоконтрастных изображений в пространственно-временной области.

Повышение контраста суммарного изображения выполнено с использованием интегрального оператора дифференцирования в дискретной форме как универсального интегрального оператора дифференцирования двумерных изображений. 
Моделированием оценены характеристики качества алгоритма. Установлено, что алгоритм

- высокоэффективен в реальной ФЦО без введения априорных данных о ее текущем состоянии в зоне контроля ОЭП: восстанавливает контрастное изображение ДО по 2-7 последовательным слабоконтрастным изображениям на $\Phi Ц К$,

- обеспечивает усиление яркости и контраста суммарного изображения ДО по 6 последовательным ФЦК соответственно до 8 и 9 раз «пропорционально» сложности фоноцелевой обстановки и слабоконтрастности изображений ДО на исходной последовательности ФЦК,

- не менее чем в 2 раза фильтрует фоновую и шумовую помехи.

Построенный алгоритм на основе изоморфного принципа, двумерного вейвлетпреобразования и интегрального оператора дифференцирования дополняет теорию и практику цифровых методов и алгоритмов обработки последовательности слабоконтрастных 2D изображений.

Алгоритм реализуем в реальном масштабе времени на современных ПЭВМ.

\section{Список литературы}

[1] Прэтт У.К. Цифровая обработка изображений. М.: Мир, 1982.

[2] Методы компьютерной обработки изображений / под ред. В.А. Сойфера. М.: Физматлит, 2003. 784 с.

[3] Гонсалес Р., Вудс Р. Цифровая обработка изображений. М.: Техносфера, 2005. 1072 c.

[4] Розенфельд А. Распознавание и обработка изображений с помощью вычислительных машин. М.: Мир, 1972. 232 с.

[5] Блаттер К. Вейвлет-анализ. Основы теории. М.: Техносфера, 2006. 272 с.

[6] Добеши И. Десять лекций по вейвлетам. Ижевск: НИЦ «Регулярная и хаотическая динамика», 2001. 464 с.

[7] Смоленцев Н.K. Основы теории вейвлетов. Вейвлеты в Matlab. M.: ДМК Пресc, 2008. 304 c.

[8] Катулев А.Н., Малевинский М.Ф. Семейство вейвлетов на основе волновой вытянутой сфероидальной функции нулевого порядка // Вестник ТвГУ. Серия: Прикладная математика. 2017. № 1. С. 71-84.

[9] Катулев А.Н., Малевинский М.Ф. Вейвлет-нечётные волновые сфероидальные функции в задаче сегментации двумерного изображения // Автометрия. 2016. T. 52, № 3. C. 10-19. https://doi.org/10.3103/S875669901603002X 
[10] Алпатов Б.А., Бабаян П.В., Балашов О.Е., Степашкин А.И Методы автоматического обнаружения и сопровождения объектов. Обработка изображений и управление. М.: Радиотехника, 2008. 176 с.

[11] Кудинов А.Н., Катулев А.Н., Малевинский М.Ф. Математические методы оценки показателей безопасности состояний динамических систем. М.: Изд-во МГУ, 2005. 375 с.

[12] Левшин В.Л. Обработка информации в оптических системах пеленгации. М.: Машиностроение, 1978. 168 с.

[13] Колмогоров А.Н., Фомин С.В. Элементы теории функций и функционального анализа. М.: Физматлит, 2004. 572 с.

\section{Образец цитирования}

Катулев А.Н., Малевинский М.Ф. Методы и алгоритмы повышения качества слабоконтрастного 2D изображения, обнаруживаемого оптико-электронным прибором динамического объекта // Вестник ТвГУ. Серия: Прикладная математика. 2018. №3. C. 95-111. https://doi.org/10.26456/vtpmk512

\section{Сведения об авторах}

\section{1. Катулев Александр Николаевич}

ведущий научный сотрудник научно-исследовательского центра Центрального научно-исследовательского института войск ВКО Минобороны России.

Россия, 170042, г. Тверь, наб. Афанасия Никитина, д. 32.

E-mail: katuleva@mail.ru

\section{2. Малевинский Михаил Федорович}

профессор кафедры математического моделирования и вычислительной математики Тверского государственного университета.

Россия, 170100, г. Тверь, ул. Желябова, д. З3, ТвГУ. 


\title{
METHODS AND ALGORITHMS FOR IMPROVING THE QUALITY OF LOW-CONTRAST 2D IMAGES DETECTED BY OPTICAL-ELECTRONIC DEVICE OF A DYNAMIC OBJECT
}

\author{
Katulev Alexander Nikolayevich \\ Leading researcher at Research Center, Central Scientific Research Institute of the \\ Aerospace Defense of the Russian Ministry of Defense \\ Russia, 170042, Tver, 32 Afanasii Nikitin quay. \\ E-mail: katuleva@mail.ru \\ Malevinskiy Mikhail Fedorovich \\ Professor at Mathematical Modeling and Computational Mathematics department, \\ Tver State University \\ Russia, 170100, Tver, 33 Zhelyabova str., TSU.
}

Received 23.01.2018, revised 05.09.2018.

\begin{abstract}
Methods and algorithms of automatic increase for the quality (brightness and contrast) of weakly contrast 2D image, being formed by optic-electronic device on detecting of the dynamical object is proposed. Brightness increase is performed by summing at frequency region on wavelet-spectra of accumulated images at the short part of time for object detection. Summing image is restored by back (reverse) wavelet conversion. Wavelet spectra are sufficient statistics, calculated by the wavelet system built on the basis of wave prolate spheroidal function of zero order. Contrast increase is carried out by integral operator of differentiation. The offered algorithm is highly effective and realized at the real time scale on modem PC.
\end{abstract}

Keywords: optic-electronic apparatus, dynamic object, weak contrast 2D image, statistic rise of brighter, algorithm, criterion of efficiency.

\section{Citation}

Katulev A.N., Malevinskiy M.F., "Methods and algorithms for improving the quality of low-contrast 2D images detected by optical-electronic device of a dynamic object", Vestnik TvGU. Seriya: Prikladnaya Matematika [Herald of Tver State University. Series: Applied Mathematics], 2018, no. 3, 95-111. (in Russian) https://doi.org/10.26456/vtpmk512

\section{References}

[1] Prett U.K., Tsifrovaya Obrabotka Izobrazhenij [Digital Image Processing], Mir Publ., Moscow, 1982 (in Russian).

[2] Metody kompyuternoj obrabotki izobrazhenij [Computer image processing methods], ed. V.A. Sojfera, Fizmatlit Publ., Moscow, 2003 (in Russian), 784 pp.

[3] Gonsales R., Vuds R., Tsifrovaya obrabotka izobrazhenij [Digital Image Processing], Tekhnosfera Publ., Moscow, 2005 (in Russian), 1072 pp. 
[4] Rozenfeld A., Raspoznavanie i obrabotka izobrazhenij s pomoshchyu vychislitelnykh mashin [Recognition and processing of images using computers], Mir Publ., Moscow, 1972 (in Russian), 232 pp.

[5] Blatter K., Vejvlet-analiz. Osnovy teorii [Wavelet analysis. Fundamentals of Theory], Tekhnosfera Publ., Moscow, 2006 (in Russian), 272 pp.

[6] Dobeshi I., Desyat lektsij po vejvletam [Ten Wavelet Lectures], Regular and Chaotic Dynamics Publ., Izhevsk, 2001 (in Russian), 464 pp.

[7] Smolentsev N.K., Osnovy teorii vejvletov. Vejvlety v Matlab, DMK Press, Moscow, 2008 (in Russian), 304 pp.

[8] Katulev A.N., Malevinskiy M.F., "A family of wavelets based on a prolate spheroidal wave function of zero order", Vestnik TvGU. Seriya: Prikladnaya Matematika [Herald of Tver State University. Series: Applied Mathematics], 2017, № 1, 71-84 (in Russian).

[9] Katulev A.N., Malevinsky M.F., "Wavelet-odd prolate spheroidal wave functions in the problem of two-dimensional image segmentation", $O p$ toelectronics, Instrumentation and Data Processing, 52:3 (2016), 223-230, https://doi.org/10.3103/S875669901603002X.

[10] Alpatov B.A., Babayan P.V., Balashov O.E., Stepashkin A.I, Metody avtomaticheskogo obnaruzheniya i soprovozhdeniya obektov. Obrabotka izobrazhenij i upravlenie [Methods for automatic detection and tracking of objects. Image Processing and Management], Radiotekhnika Publ., Moscow, 2008 (in Russian), 176 pp.

[11] Kudinov A.N., Katulev A.N., Malevinskij M.F., Mathematical methods for assessing the safety performance of states of dynamic systems, MSU Publ., Moscow, 2005 (in Russian), 375 pp.

[12] Levshin V.L., Obrabotka informatsii $v$ opticheskikh sistemakh pelengatsii [Information processing in optical direction finding systems], Mashinostroyeniye, Moscow, 1978 (in Russian), 168 pp.

[13] Kolmogorov A.N., Fomin S.V., Elementy teorii funktsij i funktsionalnogo analiza [Elements of the theory of functions and functional analysis], 7th edition, Fizmatlit Publ., Moscow, 2004 (in Russian), 572 pp. 